\title{
Comparison of Time-Delay Estimation Techniques in Acoustic Environment
}

\author{
Sanjeev Dhull \\ Asst.Professor \\ ECE DEPARTMENT \\ G.J.U.S\&T,HISAR
}

\author{
Dr Sandeep Arya \\ Chairman \\ ECE DEPARTMENT \\ G.J.U.S\&T,HISAR
}

\author{
Dr O.P Sahu \\ Assoc.Professor \\ ECE DEPARTMENT \\ N.I.T KURUKSHETRA
}

\begin{abstract}
The calculation of time delay between a signal and its echo received at a microphone has been proven to be a useful parameter. Speech enhancement, speaker localization, speech and speaker recognition are few applications of TDE methods. We are implementing various methods for the estimation of time delay. These methods are implemented in MATLAB. The reason for choosing the MATLAB as the analysis and simulation tool is that it has more flexible choices to support the simulation and is easy to do modification or data recording. These methods are crosscorrelation (CC), phase transform (PHAT). Various time-delay estimation techniques based on the cross-correlation functions are compared through simulations and measurements. Their simulation results are compared in terms of computation complexity, hardware implementation, precision, and accuracy.
\end{abstract}

\section{General Terms}

Time delay, Correlation, GCC.

\section{Keywords}

TDE,GCC,PHAT

\section{INTRODUCTION}

During last few years, the calculation of time delay[1] between a signal and its echo received at a microphone in the presence of noise is used in a number of applications like radar communications, microphone array processing and speech recognition.

The received signal at a microphone can be expressed as:

$R_{l}(t)=s(t)+n_{l}(t), \quad(1)$

$R_{2}(t)=s(t-d)+n_{2}(t), \quad(2)$

Where $R_{l}(t)$ and $R_{2}(t)$ are the output signals and $s(t)$ is the source signal, $\mathrm{n}_{1}(\mathrm{t})$ and $\mathrm{n}_{2}(\mathrm{t})$ are the two noise signals, $\mathrm{d}$ is the time delay between two signal. The signal and noise are assumed to be uncorrelated having zero-mean and Gaussian distribution. There are many algorithms to calculate the time delay (d). The crosscorrelation (CC) is a very important method for the calculation of time delay. The $\mathrm{CC}$ method cross-correlates the microphone output and takes the time argument to find the peak in the output as the estimated time delay. Another method for the calculation of time delay is phase transform (PHAT). It is also called generalized cross correlation (GCC). In this method, we improve the peak detection and the time delay estimation. We use various filter or weighting functions after the cross correlation.

\section{TDE METHODS}

In this paper we describe two methods of time delay estimation. They are explained below:
Cross-correlation:In this method we calculate the cross correlation between two noisy signals. Then locate the maximum peak in the output which gives the estimated time delay. The CC method is expressed as:

$R_{r l r 2}(\tau)=E\left[r_{1}(t) r_{2}(t-\tau)\right]$

$D c c=\arg \max \left(R_{r 1 r 2}(\tau)\right)$-------------(4)

Phase transform ( PHAT) method:It is a method to improve the estimation of time delay. It is also called generalized cross correlation (GCC). It avoids the spreading of the peak of the correlation function. The mathematical modal can be given as:

$R_{r I r 2}(\tau)=\int_{-\infty}^{\infty} \Psi(f) G_{r I r 2}(f) \exp (j 2 \pi f \tau) d f \quad(5)$

$\Psi(f)=1 / \operatorname{Gr} 1 r 2(f) \quad(6)$

$D p=\arg \max \left[R_{r I r 2}(\tau)\right]$

Where $\operatorname{Gr} 1 r 2(f)$ is the cross-spectrum of the received signal, $\Psi(f)$ is the PHAT weighting function. The simulation is carried out in simulated noisy environment. Here we take Gaussian noise for simulation and add noise to it. The two different signals are shown below. They are named as signal 1 and signal 2.The Gaussian signal [2]can be generated by using 'randn' command, which variance is one. The two signals are plotted in figure(1).

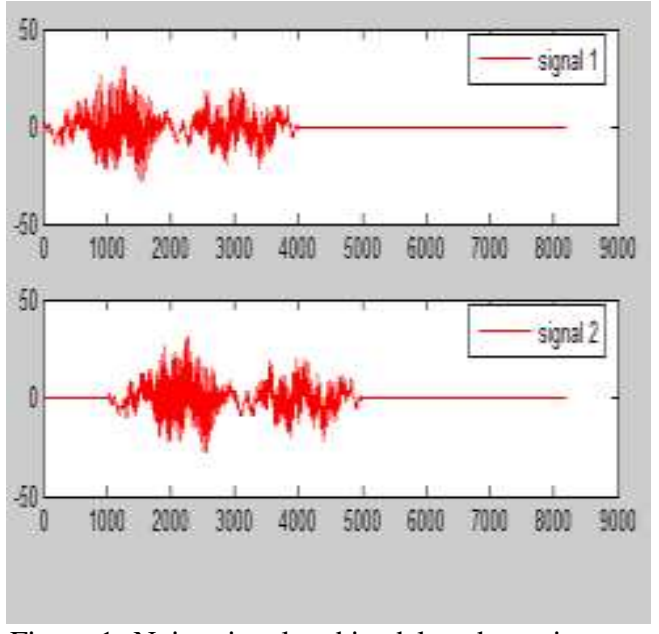

Figure 1: Noisy signal and its delayed version

Then choose the time delay as 1000T seconds, where $\mathrm{T}$ is source signal sampling period. The cross correlation result using CC and PHAT method are given in figure (2). The $\mathrm{x}$ coordinate denotes the time lag and $y$ - coordinate denotes the resulted cross-correlations. In figure 2 it is clear that peak occurs at the actual time delay i.e. $1000 \mathrm{~T}$ 


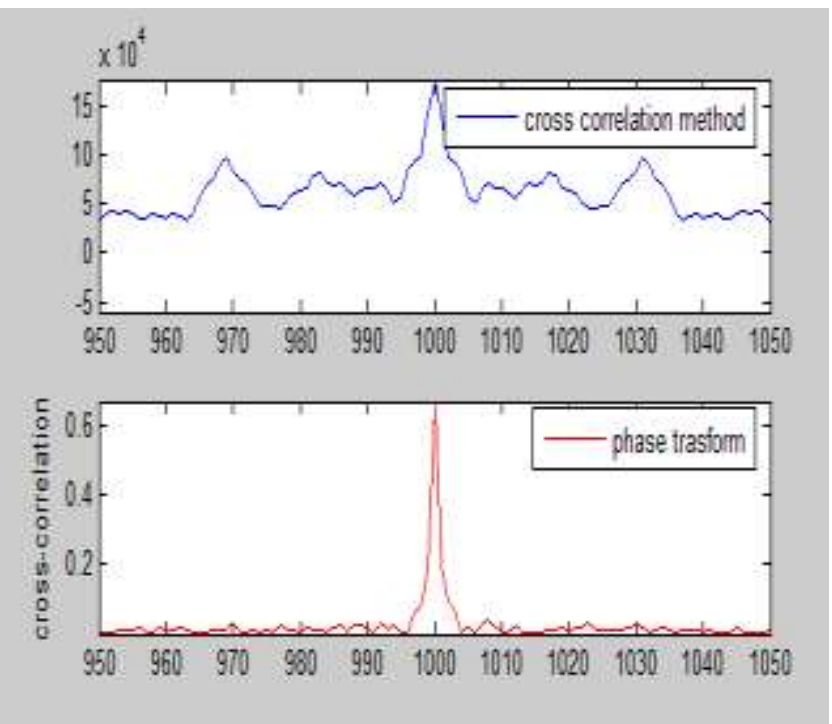

Fig2 CC, PHAT in a simulated noisy environment for $\mathrm{T}=1000$
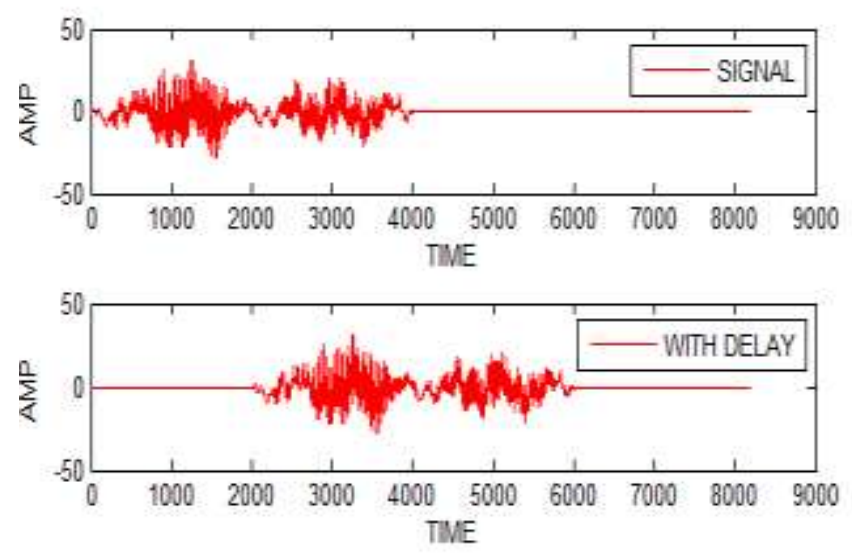

Fig.3 Noisy signal and its delayed version for $\mathrm{T}=2000$
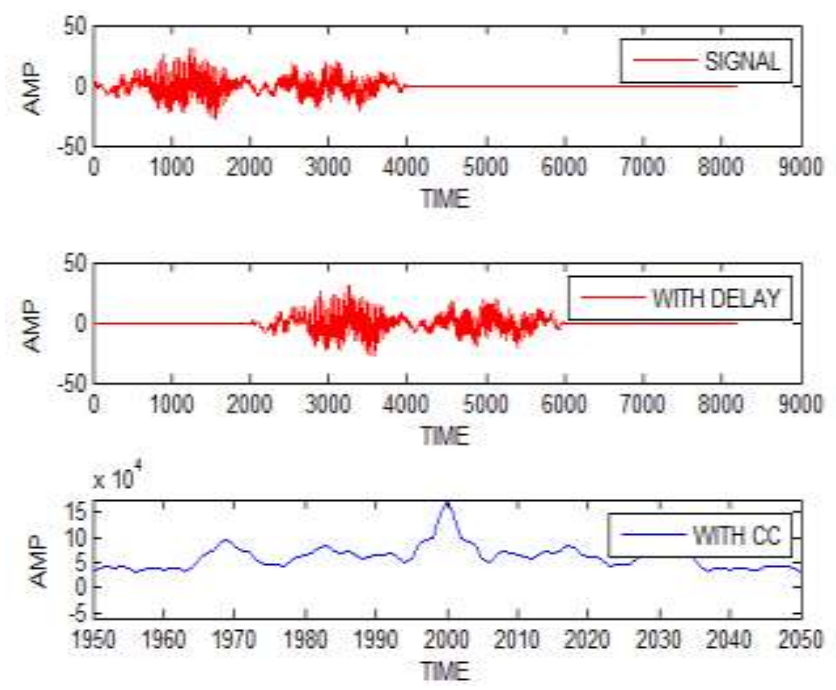

Fig.4 Delay representation by cross correlation method

The output shows that the peak
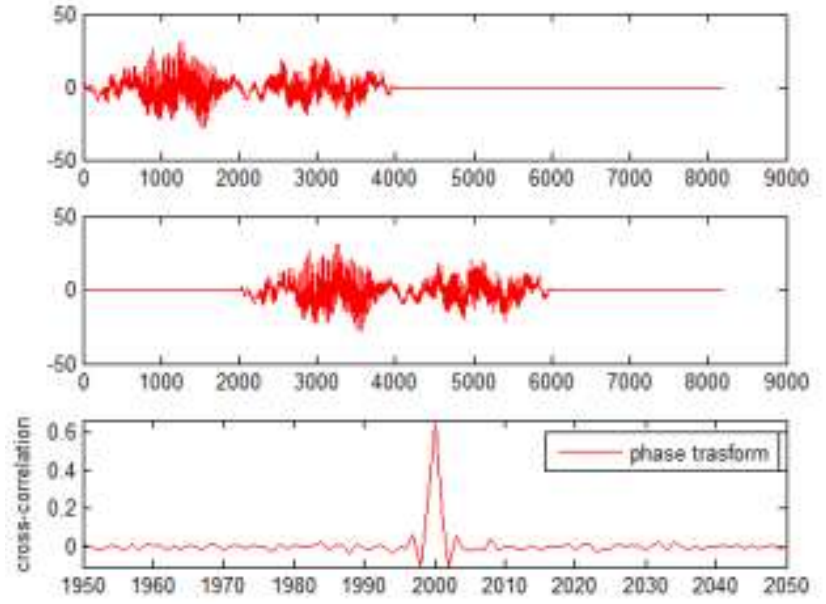

Fig5.Phase transform figure for delay representation occurs at actual time delay. Generally, room reverberation is considered as the main problem for TDE. Moreover, acoustic background noise [3]may further decrease the performance of time-delay estimators. The performance of TDE is always affected by the reverberation in a room. The problem becomes more challenging once room reverberations rise. In a highly reverberant room[4], all the known TDE methods[6] become unreliable and even fail. Few early studies have investigated the TDE problem in the presence of a few correlated additive echoes. However, the results obtained cannot be used to predict the effects of reverberation on the TDE performance since reverberation consists in the superposition of a very large number of closely spaced echoes, indeed, which are characterized by temporal and spatial correlation. In particular, the quantitative behavior of the estimator variance for reverberation can be explained naturally in terms of an equivalent signal-to-noise ratio (SNR), which treats the reverberant energy at the microphone output as undesirable noise. Namely, the high level of reverberation causes the low value of SNR. These problems in TDE can be combined into a situation where the SNR is low. There is degradation of performance at low SNR. This is often evidenced as the threshold phenomena in a plot of the variance of the estimates as a function of the SNR in TDE. These thresholds divide the SNR range into several no overlapping regions. At high SNR, this is the ambiguity-free mode of operation where differential delay estimation is subjected only to local errors. For very low SNR values, observations are dominated by noise and are essentially unhelpful for TDE.Fig3.represent the delay for $\mathrm{T}=2000$,Fig4. shows the delay estimation at $\mathrm{T}=2000$.Fig5 shows that estimation of peak is better in case of PHAT method

\section{Relation between the SNR level and the Time delay Estimation Accuracy}

In this section, the time delay is estimated under various SNR levels. The noise type is Gaussian with zero mean and variance is equal to one. To study the performance of the time delay estimation, the following experiments have been set.

The actual time delay value is set to 1000T, we calculate the time delay using CC, PHAT in different SNR situations. The various SNR level is obtained by altering the noise power. The results are plotted in Figure. The $\mathrm{x}$-coordinate presents the various SNR values, while the $y$-coordinate presents the estimated time delay. 
The plots show that the estimated time delay becomes incorrect when the SNR exceeds a certain threshold. In this case, SNR thresholds are about $-13 \mathrm{~dB}$ and $-13.5 \mathrm{~dB}$ for $\mathrm{CC}$ and PHAT respectively.

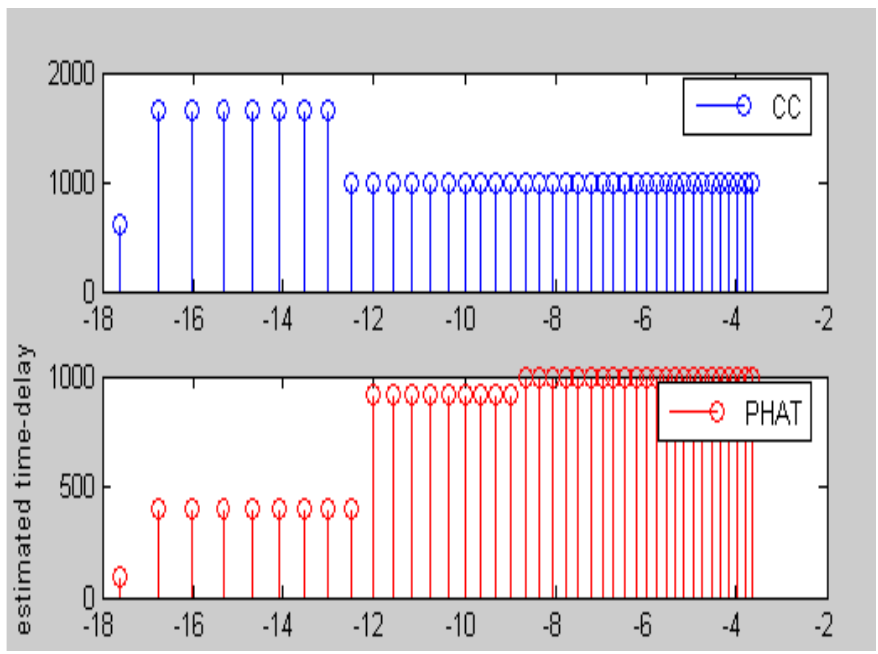

Figure 3: The relations between SNR and estimated time delay using $\mathrm{CC}$, PHAT in a simulated environment

The two TDE methods show excellent results in high SNR environments[9][10]. However, their performances become very poor when SNR is decreased. In this situation, the noisy signal becomes noise domain and a big distortion produced by the additive noise presents in the crosspower spectrum, thus the prefilter attempts to whiten the noise rather than the source signal. Each method has its own SNR threshold. Above this threshold, the estimated time delay becomes accurate and unbiased. On the other hand, the estimation method fails to function properly below the SNR threshold. This indicates that CC outperform the other TDE methods at low SNR conditions in simulated noisy environments, however PHAT has the best performance in actual environment.

\section{CONCLUSITION}

The two TDE methods are described in this paper in a simulated noisy environment. However, in the actual noisy environment, the PHAT seems to be the best choice because of its perfect performance in sharpening the correlation at the correct time delay. These methods work well in the case of high SNR. There performance decreases when the SNR is below the thresholdvalue.

\section{REFERENCES}

1 G. C. Carter: "Coherence and time delay estimation: an applied tutorial for research, development, test, and evaluation engineers", Piscataway, NJ: IEEE Press, 1993

2 Z. CH. Liang, X. ZH. Liu and Y. T. Liu: “A modified time delay estimation algorithm based on higher order statistics for signal detection problems", IEEE Signal Processing, vol.1, pp.255-258, Aug 2002

$3 \mathrm{CH}$. Zheng and TH. T. Tjeng: "A new time delay estimator based on ETDE", IEEE Transactions On Signal Processing, vol.51, no.7, pp.1859-1869, July 2003

4 S. Bédard, B. Champagne and A. Stéphenne: "Effects Of Room Reverberation On Time-Delay Estimation Performance", IEEE, Acoustics, Speech, and Signal Processing, vol.2, pp.261-264, April 1994

5 A. K. Nandi: "On the Subsample Time Delay Estimation of Narrowband Ultrasonic Echoe", IEEE Transactions on Ultrasonics, Ferroelectrics, And Frequency Control, Vol.42, N0.6, pp.993-1001, November, 1995.

6 G. C. Carter, "Time delay estimation for passive sonar signal processing,"IEEE Trans. Acoust., Speech, Signal Processing, vol.ASSP-29, no. 3, pp. 463-470, June 1981.

7 G. C. Carter, "Variance bounds for passively locating an acoustic source with a symmetric line array," J. Acoust. Soc. Amer., vol. 62,no. 4, pp. 922-926, Oct. 1977.

8 W. J. Bangs, "Array processing with generalized beamformers,"Ph.D. dissertation, Yale University, 1973.W. R. Hahn, "Optimum signal processing for passive sonar range

9 H. L. Owsley, G. R. Swope, and J. F. Law, "High resolution range estimation with a linear array," IEEE Circuits, Syst., 1982, pp. 235-240.

10 P. M. Schultheiss and J. P. Ianniello, "Optimum range and bearing estimation with randomly perturbed arrays," J. Acoust. Soc. Amer., vol. 68, no. 1 , pp. 167173, July 1990. 